\title{
EDITORIAL NOTE \\ Editorial Note on: On the feasibility of using motor imagery EEG-based brain-computer interface in chronic tetraplegics for assistive robotic arm control: a clinical test and long-term post trial follow-up
}

Spinal Cord (2012) 50, 716; doi:10.1038/sc.2012.29; published online 20 March 2012

Editorial Note on: Spinal Cord (2012) 50, 599-608; doi:10.1038/ sc.2012.14; published online 13 March 2012

Spinal cord injury is a significant source of lost productivity, such that even small incremental improvements in function and independence can significantly have an impact on quality-of-life. In order to overcome at least some of the recognized potential limitations of non-invasive brain-computer interface (BCI) systems including limited bit rate and practicality of daily use, Professor Onose and colleagues have designed a hybrid system that integrates 3D-gaze data and EEG signals from motor cortices and uses 'natural' motor imagination/intention signals. Besides the technological developments which are notable, the report is most useful and instructive because of its assessment of performance in the largest cohort to date of patients who would actually benefit from such a system (rather than healthy controls) and, perhaps more importantly, its examination of patients' long-term impressions after trialing the device. Although performance is not perfect, $\sim 80 \%$ cursor control and $\sim 30 \%$ robotic arm control are respectable, especially given the complexity of the latter task, a population that has few other options, and the non-invasive nature of the device with a no-risk trial. More importantly, the authors assess the patients' experience with the system providing insights into patients' preferences that, despite seemingly exponential growth in BCI development, are most often assumed but not formally evaluated. These devices are after all intended to improve patients' quality-oflife; if we do not specifically assess this endpoint, we will ultimately fail to accomplish our goals. In this light, although the patients in this series expressed a relatively high rate of satisfaction with few complaints with a non-invasive system, as a community, we should be cognizant of that most disabled patients may be willing to undergo invasive procedures to achieve superior BCI control. ${ }^{1}$ Future BCI studies must continue to not only assess patients' performance, but also satisfaction and impressions in order to find the best means of improving quality-of-life.

\section{CONFLICT OF INTEREST}

The author declares no conflict of interest.

N Pouratian

UCLA Neurosurgery, Los Angeles, CA, USA E-mail: npouratian@mednet.ucla.edu

1 Huggins JE, Wren PA, Gruis KL. What would brain-computer interface users want? Opinions and priorities of potential users with amyotrophic lateral sclerosis. Amyotroph Lateral Scler 2011; 12: 318-324. 